\title{
Variabilidade Climática: A Precipitação como Parâmetro de Estudo Para os Casos de Dengue no Litoral, Sertão, Serra e Sul Cearense Entre 2007 e 2019
}

\author{
Marcelo Sousa da Costa $^{1}$ (D), Roberto Alan Ferreira de Araújo ${ }^{1}$ \\ ${ }^{1}$ Centro de Ciências e Tecnologia, Mestrado Profissional em Climatologia, Universidade \\ Estadual do Ceará, Fortaleza, CE, Brasil.
}

Recebido em: 9 de Abril de 2020 - Aceito em: 17 de Maio de 2021

\begin{abstract}
Resumo
A Dengue se tornou um dos principais problemas de saúde pública no mundo tropical, especialmente, nos países com precariedade socioeconômica. Desse modo, o objetivo geral desta pesquisa é analisar e comparar os casos de Dengue com a precipitação e os fenômenos associados em quatros cidades do Estado do Ceará (Brasil): Aquiraz, Boa Viagem, Viçosa do Ceará e Brejo Santo, as quais representam ambientes geográficos distintos (litoral, sertão, serra e sul do Ceará) entre os anos de 2007 e 2019. Para isso, foi realizado um levantamento de dados estatísticos e geográficos através do Datasus, FUNCEME, IBGE, IPECE, assim como uma pesquisa descritiva dos casos de Dengue nos municípios da região, apresentando seus dados sociais, econômicos, ambientais e, principalmente, pluviométricos. Associado a estas informações, pesquisou-se sobre a influência dos mecanismos físicos e sistemas atmosféricos que influenciam as chuvas e condições climáticas na região nordeste brasileira (NEB), tais como: anomalias da Temperatura da Superfície do Mar (TSM) e o estabelecimento de ano-padrões pela técnica dos quantis com base nas precipitações dos últimos 30 anos (1990-2019). Por fim, inseriu-se os dados de precipitação e notificações de casos de Dengue na modelagem HoltWinters no software $R$, pois, percebeu-se ser o mais aplicado aos dados sazonais e de tendência observados ao longo da pesquisa. Essa ação teve como finalidade prever as precipitações e casos da doença para os próximos 24 meses, no intuito de subsidiar ações governamentais e civis para planejamento de combate à Dengue. Os resultados obtidos na pesquisa destacaram que nos anos mais secos as cidades de Boa Viagem, Viçosa do Ceará e Brejo Santo registraram maior ocorrência de casos de Dengue; para Aquiraz (cidade litorânea), os anos com volume pluviométrico próximo da normal climatológica apresentaram mais casos da doença. $\mathrm{O}$ município que registrou maior volume pluviométrico no período da pesquisa foi Viçosa do Ceará (maior altitude) que também obteve menos casos de Dengue. A cidade com menor volume pluviométrico foi Boa Viagem (sertão central cearense). Brejo Santo (maior latitude, sul do Ceará) apresentou mais ocorrências de casos de Dengue no período de 2007 a 2019. Portanto, se observou que os picos da doença nos municípios foram registrados com maior frequência em anos pouco chuvosos, exceto em Aquiraz onde foi observado aumento dos casos em anos com chuvas próximo da climatologia. No tocante aos resultados da modelagem, tais foram considerados satisfatórios, levando em consideração as tendências e sazonalidades, para prever a quantidade, principalmente, de precipitação.
\end{abstract}

Palavras-chave: precipitação, dengue, climatologia, saúde, Holt-Winters.

\section{Climate Variability: Precipitation as a Study Parameter for Dengue Cases in the Coast, Wilderness, Mountain Range and South Cearense Between 2007 and 2019}

\begin{abstract}
Dengue has become one of the main public health problems in the tropical world, especially in countries with socioeconomic precariousness. Thus, the general objective of this research is to analyze and compare the cases of dengue with precipitation and the associated phenomena in four cities in the State of Ceará (Brazil): Aquiraz, Boa Viagem, Viçosa do Ceará and Brejo Santo, in which they represent environments geographic regions (coast, backlands, mountain range and south of Ceará) between the years 2007 and 2019. For this, a survey of statistical and geographical data was
\end{abstract}

Autor da correspondência: Marcelo Sousa da Costa, marcelosousacosta@gmail.com. 
carried out through Datasus, Funceme, IBGE, IPECE, as well as a descriptive survey of dengue cases municipalities in the region, presenting their social, economic, environmental and, mainly, pluviometric data. Associated with this information, research was carried out on the influence of physical mechanisms and atmospheric systems that influence rainfall and climatic conditions in the northeast region of Brazil (NEB), such as: anomalies of the Sea Surface Temperature (TSM) and the establishment of a year -standards using the quantile technique based on rainfall over the past 30 years (1990-2019). Finally, the data of precipitation and notifications of Dengue cases were inserted in the Holt-Winters modeling in the R software, as it was perceived to be the most applied to the seasonal and trend data observed throughout the research. This action aimed to predict the precipitations and cases of the disease for the next 24 months, in order to subsidize government and civil actions for planning to combat Dengue. The results obtained in the research highlighted that in the drier years the cities of Boa Viagem, Viçosa do Ceará and Brejo Santo registered a higher occurrence of Dengue cases; for Aquiraz (a coastal city), the years with rainfall close to the climatological normal presented more cases of the disease. The municipality that recorded the highest rainfall in the survey period was Viçosa do Ceará (highest altitude), which also had fewer cases of Dengue. The city with the lowest rainfall was Boa Viagem (central backlands of Ceará). Brejo Santo (greater latitude, southern Ceará) presented more occurrences of Dengue cases in the period from 2007 to 2019. Therefore, it was observed that the peaks of the disease in the municipalities were registered more frequently in low rainy years, except in Aquiraz where it was increase in cases in years with rainfall close to climatology. Regarding the results of the modeling, these were considered satisfactory, taking into account the trends and seasonality, to predict the amount, mainly, of precipitation.

Keywords: precipitation, dengue, climatology, health, Holt-Winters.

\section{Introdução}

A relação entre a sociedade e a natureza desde muito tempo é uma fonte de investigação entre pensadores, pesquisadores e cientistas. Muitos questionamentos dessa relação já foram resolvidos, outros não. Nesse sentido, Mendonça (2007) entende que a intensificação da urbanização e a eclosão da questão ambiental tornaram evidentes os problemas sociais derivados da degradação do ambiente, tal fato exigiu dos cientistas ambientais uma maior participação na resolução da problemática, fazendo com que o clima passasse a ser abordado de um ponto de vista mais holístico.

Ribeiro et al. (2017) explica que diante das novas funções e arranjos que as cidades foram sendo estabelecidos, novos problemas de saúde foram surgindo como a dengue, pois essa doença se caracteriza como um problema recorrente dos espaços urbanos que provoca transtornos em diferentes setores da sociedade.

Barbosa (2007) afirma que as precipitações prolongadas e perenes contribuem de forma favorável na reprodução dos mosquitos e que há uma relação direta nos países tropicais entre as chuvas e a elevação no número de vetores.

Conforme o supracitado autor, a precipitação é um fator importante porque os mosquitos possuem processos aquáticos de larva e pupa. Logo, precisam de água para proliferação. Com efeito, chuvas abundantes podem propiciar surtos de dengue. Todavia, o padrão de precipitação também pode influenciar nos casos de dengue, pois os eventos de chuvas intensas e constantes podem transportar as larvas para locais distantes da proliferação ou ainda exterminá-las. Em contrapartida, chuvas menos intensas e inconstantes podem fazer surgir criadouros e manter um ambiente propício para a dispersão e sobrevivência dos mosquitos.
Ribeiro et al. (2017) afirma que a dengue possui como agente etiológico um arbovírus que é transmitido por artrópodes, cujo principal transmissor é o Aedes aegypti, que ocorre quando o mosquito se contamina ao picar uma pessoa doente, permanecendo com o vírus durante o período vital, daí o mosquito infectado pica uma pessoa saudável para alimentar-se e injeta o vírus com sua saliva.

Sylvestre (2014) alerta que a Dengue se configura como um dos maiores problemas de saúde pública da atualidade, pois mais da metade da população mundial encontra-se em áreas de risco de transmissão da doença.

A Dengue é uma doença de característica sazonal. Contudo, devido à dimensão continental do país e suas diferentes zonas climáticas, os auges de transmissão diferem no tempo e entre as regiões (Teixeira, 2014). Os níveis da doença são tão alarmantes que cerca de três milhões de casos foram notificados no Brasil nos últimos cinco anos (Luz, 2014).

No Brasil, até novembro de 2020 foram registrados 971.136 casos de Dengue. (Brasil, 2020). Os casos confirmados de Dengue no Ceará cresceram aproximadamente $20 \%$ em comparação ao ano de 2019 , conforme dados epidemiológicos da secretaria de saúde do estado. Na região de Fortaleza o aumento foi de $65 \%$ e na região do Cariri mais de $100 \%$ de aumento.

Portanto, o objetivo dessa pesquisa é verificar a variabilidade dos casos de Dengue em diferentes geoambientes no Estado do Ceará, utilizando dados quantitativos e modelagem estatística associada à análise de séries temporais. Ao passo que os objetivos específicos são: identificar o padrão de sazonalidade das chuvas e dos casos de dengue; verificar e relacionar a influência dos sistemas atmosféricos e mecanismos físicos como El Niño Oscilação Sul (ENSO) e anomalias de temperatura da superfície do mar (TSM) no Atlântico Tropical, indicativos de dipo- 
los de TSM que influenciam a variabilidade de precipitação nessa área do Nordeste; propor e prever um modelo que colabore nas estimativas das precipitações e casos de Dengue para os próximos dois anos (2020-2021) através do modelo Holt-Winters Aditivo utilizando a linguagem R.

Assim, esta pesquisa analisará a relação entre os aspectos climáticos, especificamente, as chuvas e os casos de Dengue levando em consideração as diferentes regiões geográficas do Ceará: litoral, sertão, serra e sul, na busca de responder as seguintes indagações: qual o grau de correlação e significância dos elementos climáticos com a dengue? Diferentes características geoambientais têm alguma relevância nesse processo?

\section{Materiais e Métodos}

\section{1 Área de estudo}

Para a realização desta pesquisa, estudou-se quatro cidades do estado do Ceará (Fig. 1) com características populacionais próximas, mas que se encontram em diferentes geoambientes: litoral representado por Aquiraz, onde recebe mais influência de diversos sistemas atmosféricos como as brisas marítimas; é uma área de planície com clima quente sub-úmido, pluviosidade e temperatura média de: $1.379,9 \mathrm{~mm}$ e $26{ }^{\circ} \mathrm{C}$ e $28{ }^{\circ} \mathrm{C}$, baixa amplitude térmica e concentração de chuvas de janeiro a maio (Ipece, 2019).

Já Boa Viagem (Fig. 1) se localiza no sertão do Ceará, na chamada depressão sertaneja, onde a formação geológica é escudo cristalino, na qual se caracteriza por baixa impermeabilidade das rochas, apresenta clima tropical semiárido, pluviosidade média de $703,8 \mathrm{~mm}$, temperaturas entre $26^{\circ} \mathrm{C}$ a $28^{\circ} \mathrm{C}$ e período chuvoso de fevereiro a abril (Op. Cit.).

Viçosa do Ceará (Fig. 1) está situada na Chapada da Ibiapaba com uma altitude de $685 \mathrm{~m}$, clima quente subúmido, pluviosidade média de $1.349 \mathrm{~mm}$, temperatura média entre $22{ }^{\circ} \mathrm{C}$ e $24{ }^{\circ} \mathrm{C}$ com período chuvoso de janeiro a abril (Ipece, 2019).

Brejo Santo que se encontra ao sul do Ceará (Fig. 1) e no sopé da Chapada do Araripe, sofre mais o efeito da continentalidade entre as cidades; possui clima tropical quente brando e semiárido com temperaturas entre $24^{\circ} \mathrm{C} \mathrm{e}$ $26{ }^{\circ} \mathrm{C}$, pluviosidade média de $895,8 \mathrm{~mm}$ (Op. Cit.).

\subsection{Dados}

Para compor as bases deste estudo foi feito o levantamento de dados estatísticos e geográficos através do Departamento de Informática do Sistema Único de Saúde do Brasil - DATASUS; da Fundação Cearense de Meteorologia e Recursos Hídricos - FUNCEME; do Instituto Nacional de Pesquisas Espaciais - INPE; do Instituto Bra-

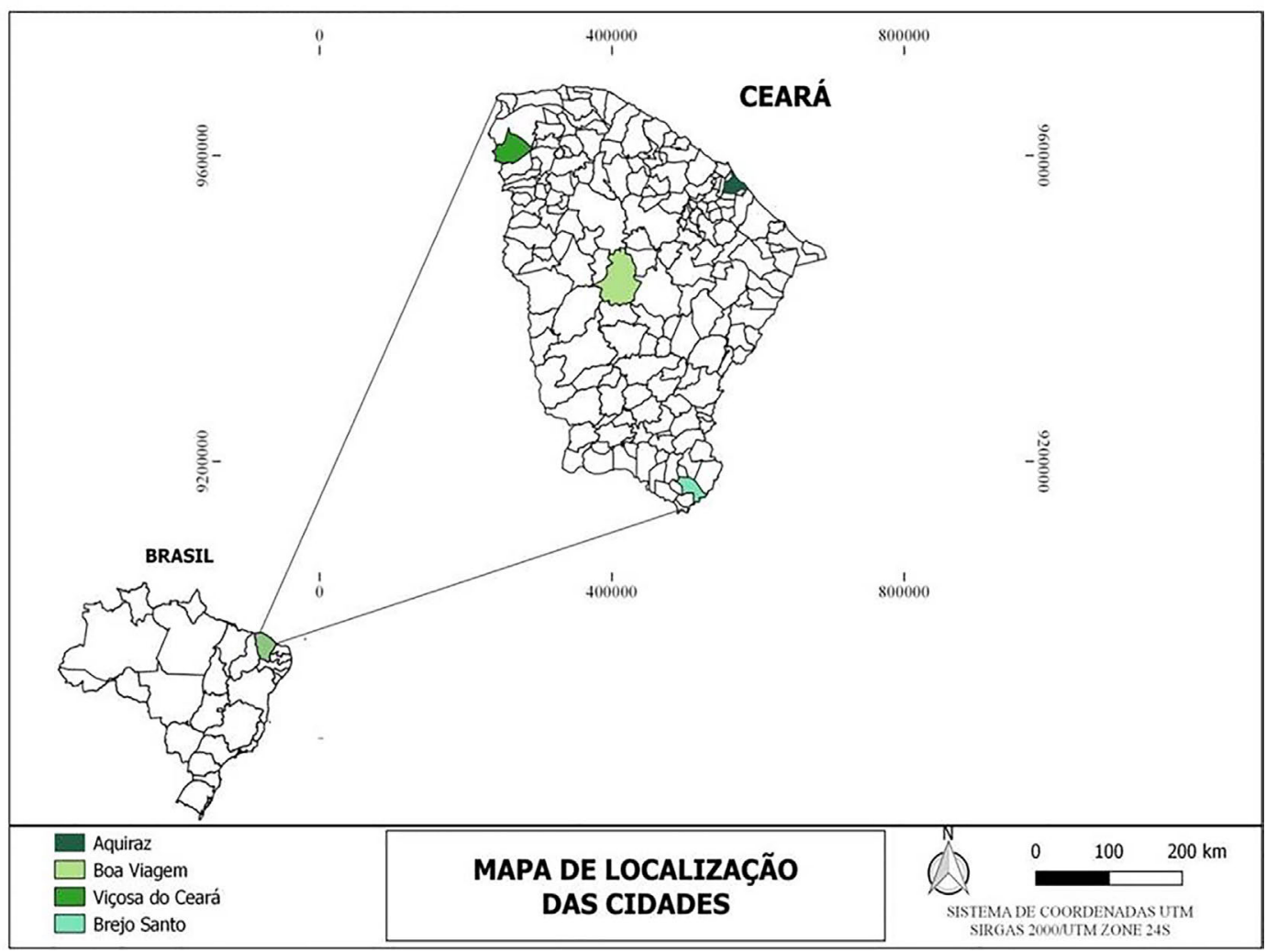

Figura 1 - Mapa de Localização das cidades. 
sileiro de Geografia e Estatística - IBGE; do Instituto de Pesquisa e Estratégica Econômica do Ceará - IPECE, bem como uma pesquisa descritiva dos casos de Dengue nos municípios da região.

As informações dos casos de Dengue e precipitações foram cruzadas e representadas por gráficos de colunas (precipitação) e linhas (casos de Dengue), com o fim de fornecer uma análise acurada para cada cidade e de todos os anos entre 2007 e 2019.

Relacionaram-se as supracitadas análises com os fenômenos atmosféricos atuantes no NEB, observando os meses com maiores e menores quantidade de casos de Dengue e volume de precipitação, e ao final de cada período anual foi estabelecida uma comparação entre as quatro cidades na busca de identificar padrões e correlações significativas.

A partir da metodologia de Pinkayan, mas aplicada por Xavier (2004) no Brasil foi estabelecida a padronização dos anos: muito seco, seco, normal, chuvoso e muito chuvoso, com a utilização da técnica estatística dos quantis. Desse modo, foi analisado o comportamento da chuva em uma série histórica de 30 anos (1990-2019). Com os anos-padrões caracterizados, especificou-se a análise para os treze anos da série da pesquisa de cada cidade para correlacionar com os casos de Dengue e total pluviométrico anual.

A pesquisa foi finalizada com a aplicação da modelagem Holt-Winters Aditivo por meio da linguagem R no ambiente RStudio para analisar o comportamento das variáveis precipitação e Dengue e as suas previsibilidades.

O R é um software livre para o desenvolvimento de procedimentos estatísticos e gráficos no computador como modelagem, testes estatísticos e análise de séries temporais. O RStudio é um produto de código aberto que está disponível pública e gratuitamente; integra-se ao ambiente $\mathrm{R}$ que inclui edição de texto e pode salvar objetos no computador bem como fazer download de pacotes (Olivoto e Sari, 2021).

Banditvilai e Anansatitzin (2017) ressaltam que o método do Holt-Winters estima três parâmetros de suavização, associados ao nível, tendência e fatores sazonais. Este método é amplamente utilizado e provou realizar uma boa previsão em dados de séries temporais com tendência linear e comportamento sazonal.

Shamsuddin et al. (2016) afirma que:

O método Holt-Winters é usado para gerenciar séries temporais quando há tendências e variações sazonais. Existem duas formas do método Holt-Winters: multiplicativa e aditiva, cuja utilização depende das características das séries temporais específica.

Por isso escolheu-se esse modelo para esta pesquisa, uma vez que os dados possuem tendência e sazonalidade, o que possibilita a previsão adequada através da sua utilização.

As equações do modelo estão escritas a seguir:

$$
\begin{aligned}
& \bar{Y}_{-}(t+n)=E_{t}+n T_{T}+S_{t+n+p} \\
& E_{t}=\propto\left(Y_{t}-S_{t-p}\right)+(1-\propto)\left(E_{t-1}+T_{t-1}\right) \\
& T_{1}=\beta\left(E_{t}-E_{t-1}\right)+(1-\beta) T_{t-1} \\
& S_{t}=\gamma\left(Y_{t}-E_{t-1}\right)+(1-\gamma) S_{t-p} \\
& 0 \leq \propto, \beta \text { e } \gamma \leq 1
\end{aligned}
$$

onde $Y_{t}=$ valor observado em uma série temporal; $\bar{\Upsilon}_{t=n}=$ valor estimado para o período $n$, a partir de um valor observado; $E_{t}=$ valor do nível observado excluído da tendência; $S_{t}=$ coeficiente de sazonalidade para o período; $P=$ número de períodos sazonais presentes nos dados; $\propto$, $\beta$ e $\gamma=$ parâmetros de suavização.

Assim, os dados das precipitações e casos de Dengue das quatro cidades no período de 2007 a 2019 foram inseridos no software R, no ambiente Rstudio e depois realizou-se a aplicação no modelo Holt-Winters Aditivo, pois se compreendeu ser o mais adequado para prever as variáveis da pesquisa que possuíram um grau de tendência e sazonalidade durante as análises.

\section{Resultados e Discussões}

Através da Tabela 1, verifica-se que nos últimos 13 anos, Aquiraz apresentou 6 anos com índices pluviométricos dentro da normalidade, 3 anos com anos muito chuvosos e 4 anos secos ou muito secos. Os anos que apresentaram mais casos de Dengue foram em 2015 e 2018, ambos os períodos com precipitações dentro do normal, já os anos com menores quantidades de casos de Dengue ocorreram em 2010 (seco) e 2019 (muito chuvoso).

A caracterização pluviométrica de Boa Viagem entre os anos de 2007 a 2019 foram: 2 anos dentro do normal, 5 anos como chuvosos ou muito chuvosos e 6 anos secos ou muito secos. No tocante aos índices da doença, as maiores quantidades de casos de Dengue foram em 2013 e 2015, anos muito seco e seco. Já os anos com menores registros da doença aconteceram em 2007 e 2018, considerados anos normal e chuvoso (Tabela 1).

Os dados pluviométricos de Viçosa do Ceará são: 1 ano normal, 6 anos chuvosos ou muito chuvosos e 6 anos secos ou muito secos. Os anos com maiores registros da Dengue ocorreram em anos considerados secos: 2015 e 2016, em contrapartida os anos com menos casos de Dengue foram em 2008 e 2018, muito chuvoso e chuvoso, respectivamente (Tabela 1).

Em Brejo Santo, a pluviometria ocorreu da seguinte forma: 05 anos normais, 3 anos muito chuvosos e 5 anos secos ou muito secos. Desse modo, os anos com maiores registros da doença foram 2007, 2015 e 2017, todos os anos secos, já os anos com menores ocorrências foram 2008 e 2009 , muito chuvoso e normal, respectivamente (Tabela 1).

A Fig. 2 mostra a relação anual entre casos de Dengue e precipitação de Aquiraz entre 2007 a 2019. Assim, percebe-se que os anos de 2015 e 2018 apresentaram 
maiores casos de Dengue e não, necessariamente, foram os anos mais chuvosos. Inclusive, 2019 foi considerado

Tabela 1 - Comparação da caracterização da precipitação das cidades.

\begin{tabular}{|c|c|c|c|c|c|}
\hline \multicolumn{6}{|c|}{ Comparação das cidades } \\
\hline Ano & Aquiraz & $\begin{array}{l}\text { Boa Via- } \\
\text { gem }\end{array}$ & $\begin{array}{l}\text { Viçosa do } \\
\text { Ceará }\end{array}$ & $\begin{array}{l}\text { Brejo } \\
\text { Santo }\end{array}$ & Anomalia \\
\hline 2007 & Normal" & Normal & $\begin{array}{l}\text { Muito } \\
\text { Seco }\end{array}$ & Seco & $\begin{array}{c}\text { El Niño } \\
\text { Fraco }\end{array}$ \\
\hline 2008 & Normal & $\begin{array}{c}\text { Muito } \\
\text { Chuvoso }\end{array}$ & Chuvoso & $\begin{array}{c}\text { Muito } \\
\text { Chuvoso" }\end{array}$ & $\begin{array}{l}\text { La Niña } \\
\text { Forte }\end{array}$ \\
\hline 2009 & $\begin{array}{c}\text { Muito } \\
\text { Chuvoso }\end{array}$ & $\begin{array}{c}\text { Muito } \\
\text { Chuvoso }\end{array}$ & $\begin{array}{c}\text { Muito } \\
\text { Chuvoso }\end{array}$ & Normal & $\begin{array}{c}\text { La Niña } \\
\text { Fraco }\end{array}$ \\
\hline 2010 & Seco & Seco & Normal & Normal & $\begin{array}{l}\text { El Niño } \\
\text { Moderado }\end{array}$ \\
\hline 2011 & $\begin{array}{c}\text { Muito } \\
\text { Chuvoso }\end{array}$ & $\begin{array}{c}\text { Muito } \\
\text { Chuvoso }\end{array}$ & Chuvoso & $\begin{array}{c}\text { Muito } \\
\text { Chuvoso }\end{array}$ & $\begin{array}{c}\text { La Niña } \\
\text { Forte }\end{array}$ \\
\hline 2012 & $\begin{array}{l}\text { Muito } \\
\text { Seco }\end{array}$ & $\begin{array}{l}\text { Muito } \\
\text { Seco }\end{array}$ & $\begin{array}{l}\text { Muito } \\
\text { Seco }\end{array}$ & $\begin{array}{c}\text { Muito } \\
\text { Seco }\end{array}$ & $\begin{array}{l}\text { La Niña } \\
\text { Moderado }\end{array}$ \\
\hline 2013 & $\begin{array}{l}\text { Muito } \\
\text { Seco }\end{array}$ & $\begin{array}{l}\text { Muito } \\
\text { Seco" }\end{array}$ & Seco & Normal & $\begin{array}{c}\text { Sem Anom- } \\
\text { alia }\end{array}$ \\
\hline 2014 & Seco & $\begin{array}{l}\text { Muito } \\
\text { Seco }\end{array}$ & $\begin{array}{l}\text { Muito } \\
\text { Seco }\end{array}$ & Normal & $\begin{array}{c}\text { Sem Anom- } \\
\text { alia }\end{array}$ \\
\hline 2015 & Normal" & Seco & Seco" & Seco" & $\begin{array}{c}\text { El Niño } \\
\text { Fraco }\end{array}$ \\
\hline 2016 & Normal & Seco & Seco" & $\begin{array}{l}\text { Muito } \\
\text { Seco }\end{array}$ & $\begin{array}{l}\text { El Niño } \\
\text { Muito Forte }\end{array}$ \\
\hline 2017 & Normal & Normal & Chuvoso & Seco & $\begin{array}{c}\text { La Niña } \\
\text { Fraca }\end{array}$ \\
\hline 2018 & Normal $^{* *}$ & Chuvoso" & Chuvoso & $\begin{array}{c}\text { Muito } \\
\text { Chuvoso }\end{array}$ & $\begin{array}{c}\text { La Niña } \\
\text { Fraca }\end{array}$ \\
\hline 2019 & $\begin{array}{c}\text { Muito } \\
\text { Chuvoso }\end{array}$ & $\begin{array}{c}\text { Muito } \\
\text { Chuvoso }\end{array}$ & Chuvoso & Normal & $\begin{array}{c}\text { El Niño } \\
\text { Fraco }\end{array}$ \\
\hline
\end{tabular}

Ano com menor quantidade de casos de dengue no município.

"Ano com maior quantidade de casos de dengue no município. um ano muito chuvoso, contudo, foi um dos anos com menor quantidade de casos da doença. Assim, pode-se inferir que uma quantidade extrema de chuva pode levar ao não desenvolvimento dos criadouros do mosquito no ambiente litorâneo, visto que as maiores quantidades de notificações ocorreram em anos com chuvas dentro da normalidade.

Em Boa Viagem, conforme a Fig. 3 pode-se perceber que os anos de pico da doença foram 2008, 2011, 2013 e 2015.

Os anos com maiores notificações da doença foram em 2013 e 2015, considerados muito seco e seco. Portanto, para cidade do sertão cearense, os anos com chuvas abaixo da média histórica causaram mais casos da doença que os anos chuvosos (Fig. 3).

Conforme Fig. 4, os casos de Dengue em Viçosa do Ceará, cidade com maior altitude dentre as pesquisadas, apresentou durante todos os anos baixo número de notificações da doença. Assim, nota-se que o ano de 2015 foi o ano com maior registro de casos, seguido por 2016, ambos os anos considerados secos.

Na cidade que fica no sopé da Chapada do Araripe, os anos com maiores registros da doença foram 2007, 2015 e 2017, todos os anos considerados como anos secos (Fig. 5).

Desse modo, verificou-se que apenas na cidade litorânea, os casos de Dengue apresentaram maior quantidade em anos considerados normais em relação à pluviosidade, nas demais cidades que representam a serra, sertão e sul do Ceará, foram os anos secos que houve mais notificações da doença. Já os anos normais, chuvoso ou muito chuvoso foram os anos com menor quantidade de notificações para as quatro cidades da pesquisa.

Com os resultados obtidos, pode-se concluir que outras variáveis podem explicar a sazonalidade da Dengue

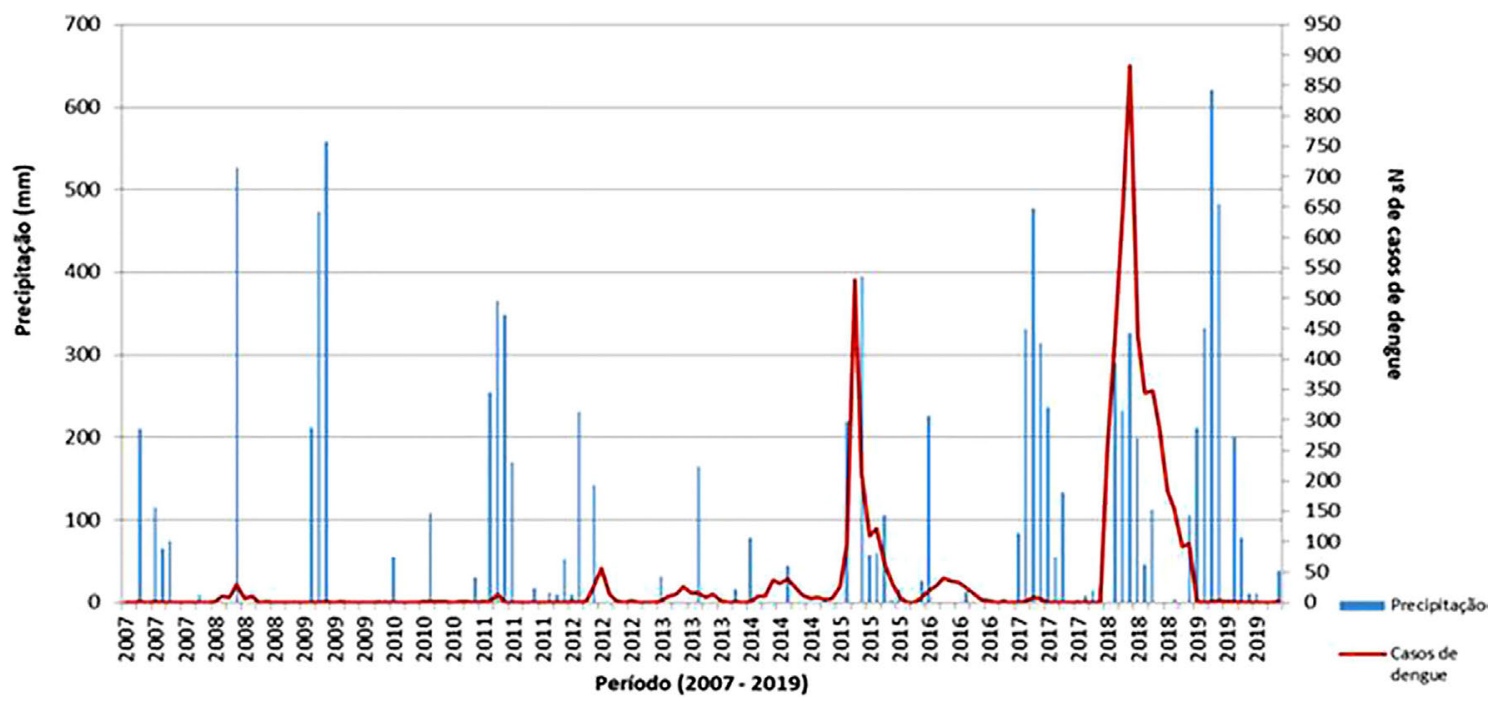

Figura 2 - Aquiraz, relação entre precipitação e casos de dengue (2007-2019). 


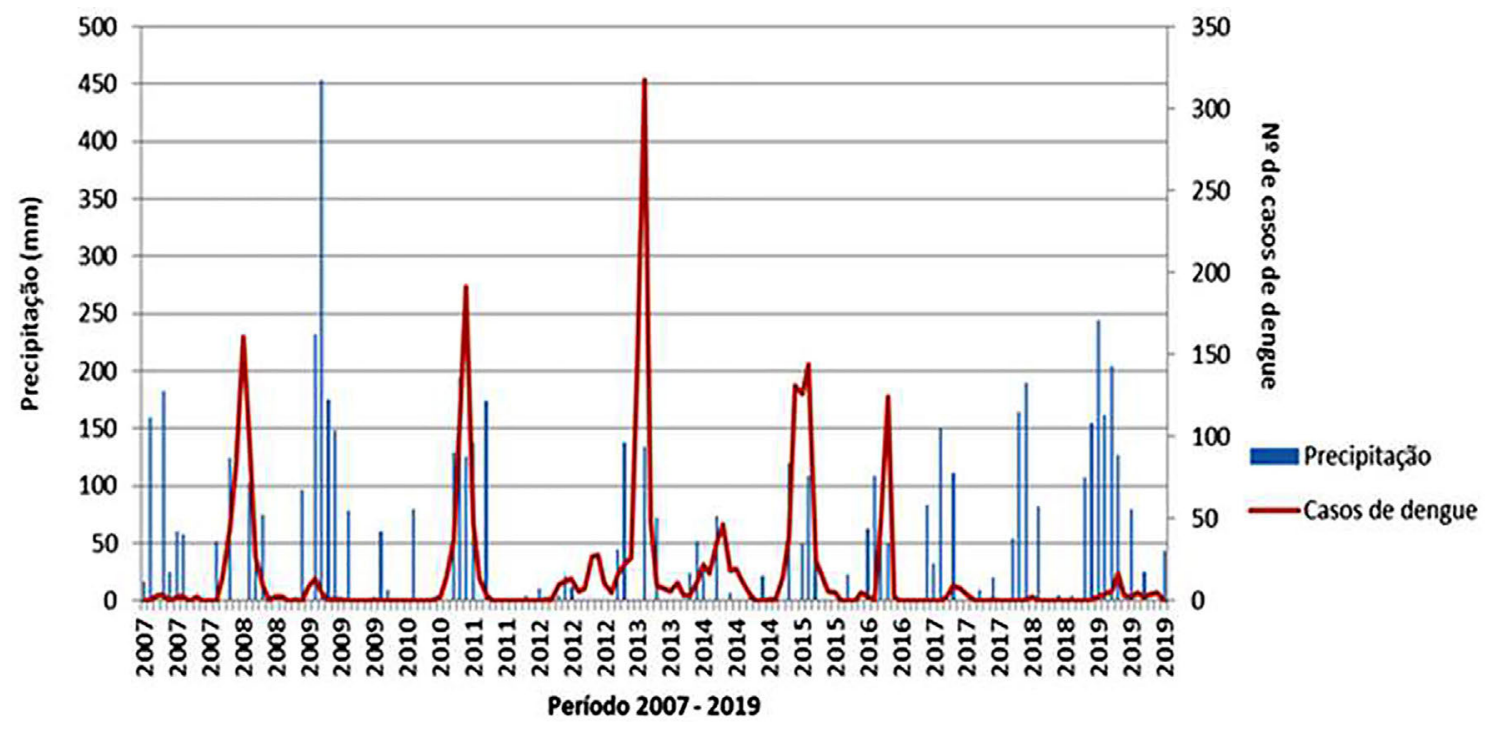

Figura 3 - Boa Viagem, relação entre precipitação e casos de dengue (2007-2019).

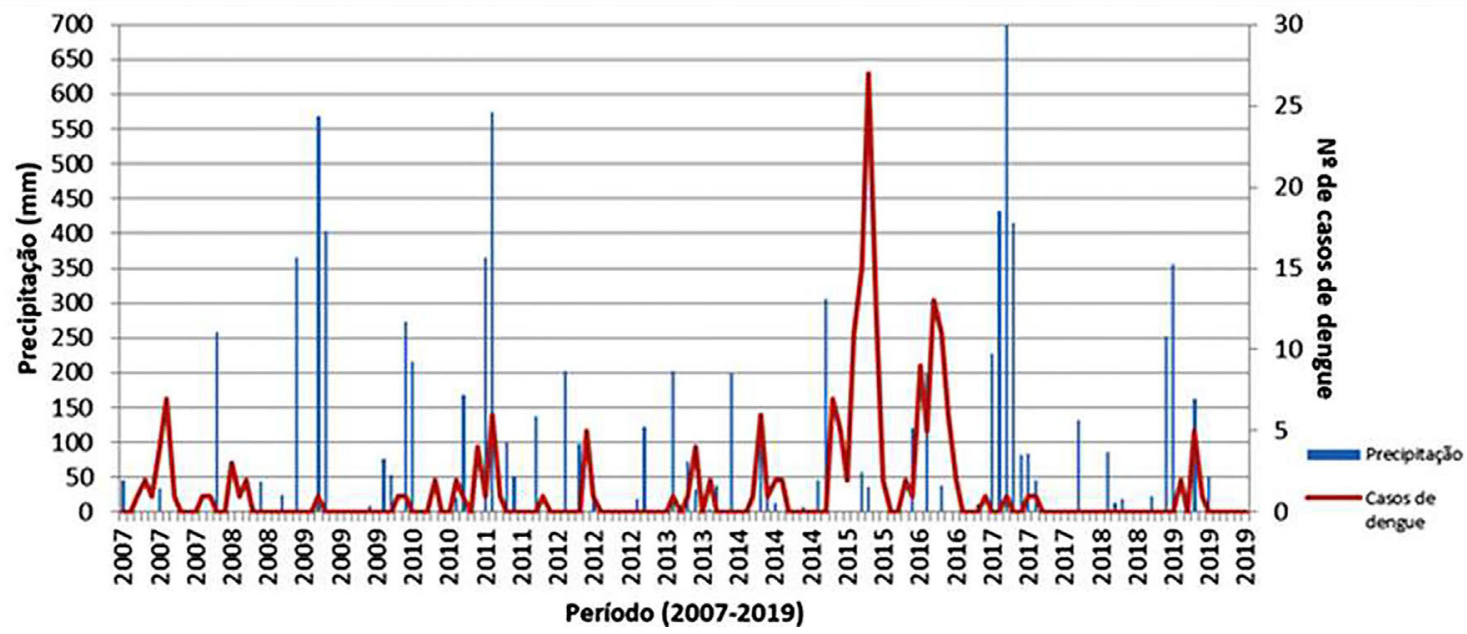

Figura 4 - Viçosa do Ceará, relação entre precipitação e casos de dengue (2007-2019).

nas cidades como a temperatura, verificada por Araújo (2012) que analisou e validou modelos matemáticos/estocásticos na observação de risco de epidemia por vetores, com enfoque na Dengue, na qual investigou a dinâmica da transmissão e os eventos probabilísticos. Assim, os resultados do modelo sugeriram uma forte dependência do inseto com as variáveis climáticas, especialmente, temperatura, e um período de risco de epidemia entre o final de dezembro e o início de maio para as cidades do Estado da Paraíba.

Ao passo que variáveis sociais também podem explicar os casos de Dengue, como Magalhães (2014) ao estudar o comportamento espaço-temporal da Dengue e sua relação com os elementos atmosféricos e socioeconômicos em Fortaleza/CE, o autor apontou que as correlações entre as variáveis epidemiológicas e socioeconômi- cas são mais fortes nos meses de início das epidemias, já as variáveis Renda Média de Moradores por Domicílio e Porcentagem de Domicílios Ligados à Rede Geral de Esgoto ou Pluvial foram as variáveis que mais se correlacionaram com a incidência da doença. Assim, a autocorrelação espacial apontou os bairros de Fortaleza com piores condições de saneamento como aqueles com elevada incidência no início dos episódios epidêmicos.

Ainda sobre a relação da Dengue e condições sociais, Silva (2019) verificou a relação entre a climatologia, as condições sanitárias (lixo) e a ocorrência de arbovirose (Dengue e Chinkungunya) em Quixadá no Ceará entre 2016 a 2019. De acordo com o estudo, a condição sanitária da cidade foi a variável correlata principal para o quadro de notificações da Dengue e Chinkungunya no período, uma vez que o aumento nos índices pluviomé- 


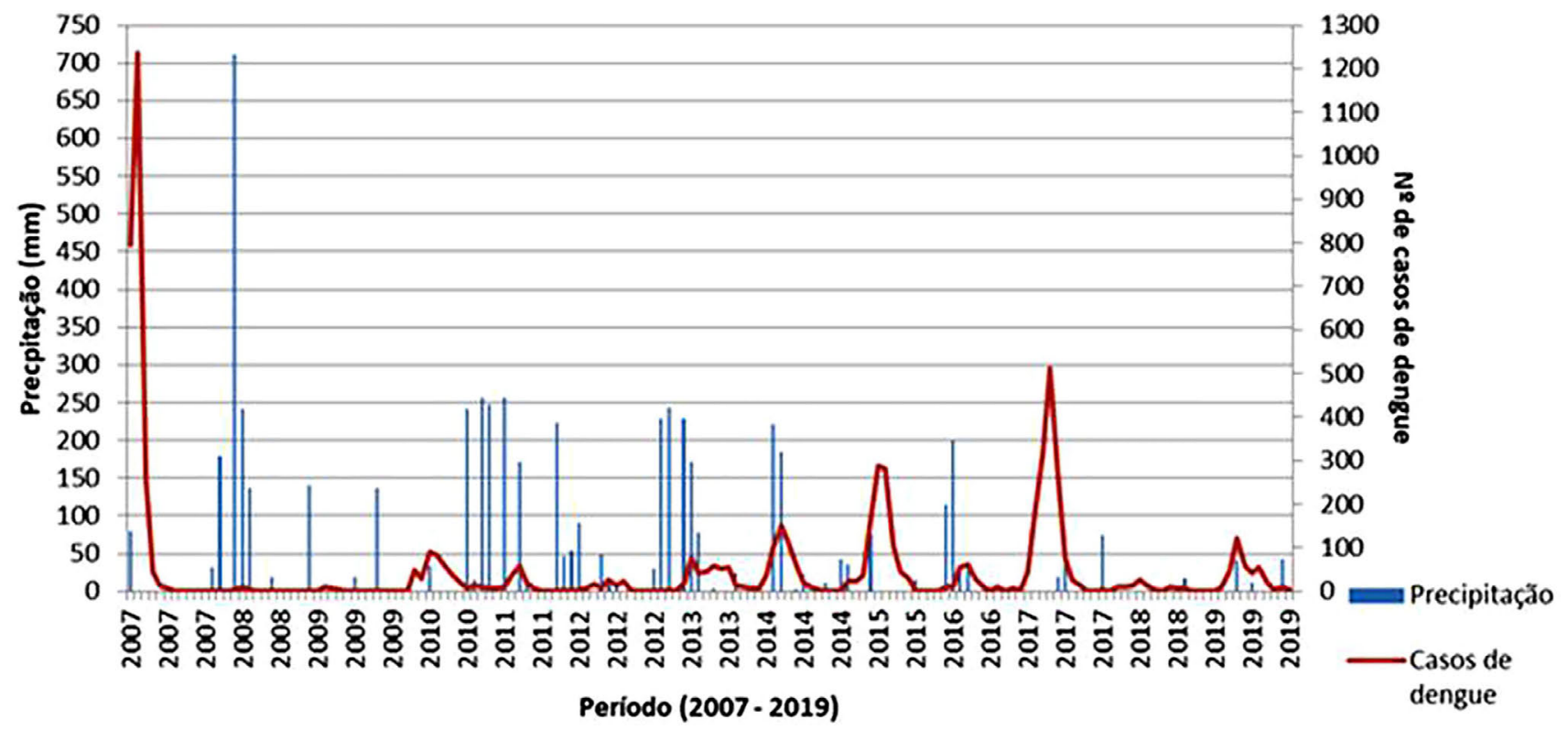

Figura 5 - Brejo Santo, relação entre precipitação e casos de dengue (2007-2019).

tricos não resultou na elevação dos casos, entretanto, quando a situação sanitária precária foi mitigada, os registros da doença diminuíram, portanto, houve uma relação diretamente proporcional entre casos confirmados das arboviroses e o acúmulo de lixo.

Desse modo, a análise conjunta de variáveis atmosféricas, econômicas e socioambientais ajudará a propor ferramentas eficientes para a prevenção e controle dos casos de Dengue.

\subsection{Aplicação da modelagem}

Cada cidade da pesquisa teve a previsão das precipitações e dos casos de dengue para os 24 meses subsequentes ao período do estudo, ou seja, as previsões foram para 2020 e 2021.

\subsubsection{Aquiraz}

A decomposição da série: observado, tendência, sazonalidade e ruídos aleatórios (Figura 6-A) mostrou que no período de 2008 a 2009 há picos de precipitações que são os ruídos aleatórios. Entre 2012 a 2015 ocorre uma baixa nas precipitações, e uma tendência de crescimento nas precipitações a partir de 2016.

Percebe-se que a previsão (Fig. 6-B) seguiu a tendência dos anos anteriores, subestimando alguns valores, todavia, com resultados dentro do esperado, levando em consideração o período histórico da cidade.

A previsão para os casos de Dengue na cidade de Aquiraz (Fig. 6-D) apresentou valores baixos, muito próximos dos anos de 2007 a 2015. Contudo, o erro residual foi grande devido os picos em 2015 e 2018 fruto da excepcionalidade do ano anterior.

\subsubsection{Boa Viagem}

Percebeu-se uma sazonalidade nas precipitações e uma irregularidade na quantidade de chuvas nos primeiros anos da pesquisa, depois uma queda na quantidade de chuvas a partir de 2012 até 2016 , e deste último uma tendência ao aumento no volume das precipitações (Fig.7-A).

Observa-se que a previsão da precipitação (Fig. 7-B) revelou valores próximos aos anos imediatamente anteriores, portanto, seguiu a tendência de aumento no volume pluviométrico.

Quanto aos casos de dengue (Fig. 7-C) ocorre uma queda nas notificações a partir de 2016. Esse é um resultado interessante, pois comprova os dados da pesquisa, na qual os anos mais secos (2012-2016) foram os anos com maiores registros de casos de Dengue.

A relação entre o observado e ajustado em Boa Viagem apresentou valores discrepantes devidos as oscilações dos casos de dengue, assim, o modelo não conseguiu captar satisfatoriamente essas ondulações dos dados no período da pesquisa.

Concernente à previsão (Fig. 7-D), nota-se que o modelo previu um suave aumento em relação aos anos anteriores, levando em consideração, portanto, os picos de casos no período da pesquisa. Já o erro residual teve valores negativos significativos entre 2009 a 2015, período que abarca os picos de casos do estudo.

\subsubsection{Viçosa do Ceará}

A previsão de precipitação seguiu a tendência de 2018 e 2019 com chuvas entre a caracterização de normal e chuvoso. 
A - Precipitação: decomposição da série temporal

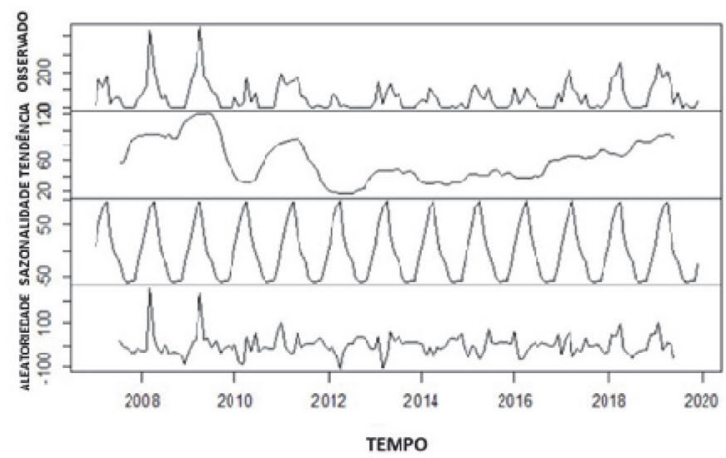

C - Dengue: decomposição da série temporal

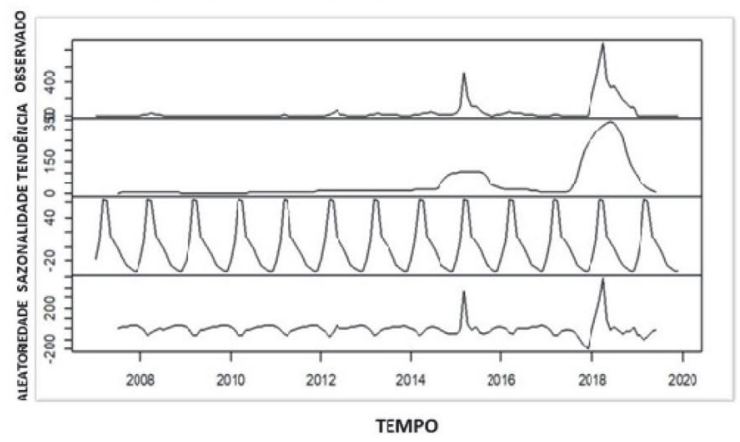

Figura 6 - Resultados da modelagem para Aquiraz (2007-2019).

A - Precipitação: decomposição da série temporal

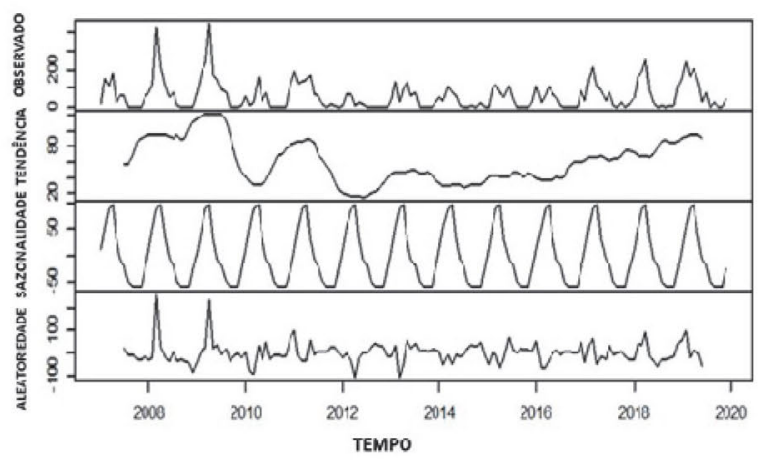

C - Dengue: decomposição da série temporal

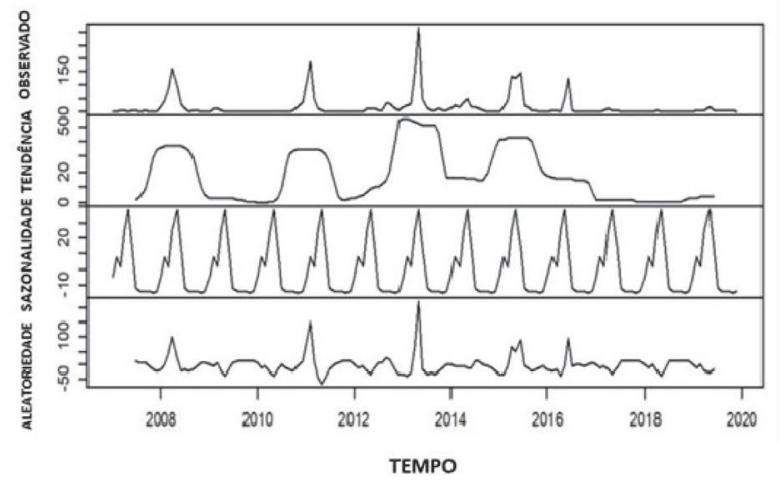

Figura 7 - Resultados da modelagem para Boa Viagem (2007-2019).
B - Precipitação: previsão Holt-Winters Aditivo

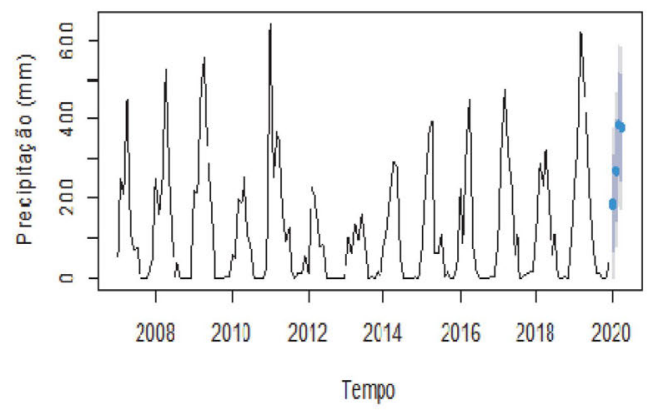

D - Dengue: previsão Holt-Winters Aditivo

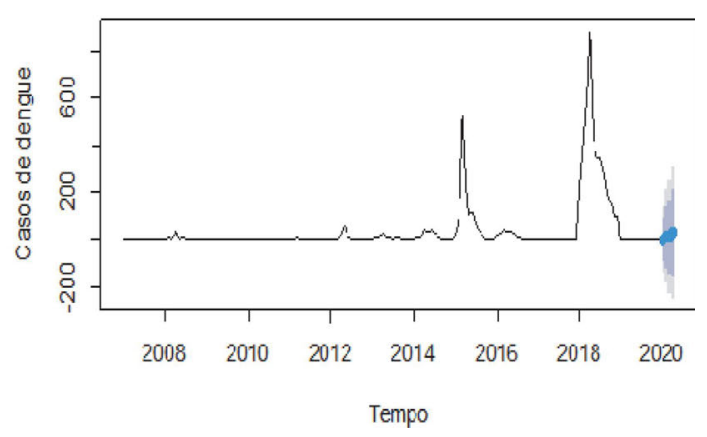

B - Precipitação: previsão Holt-Winters Aditivo

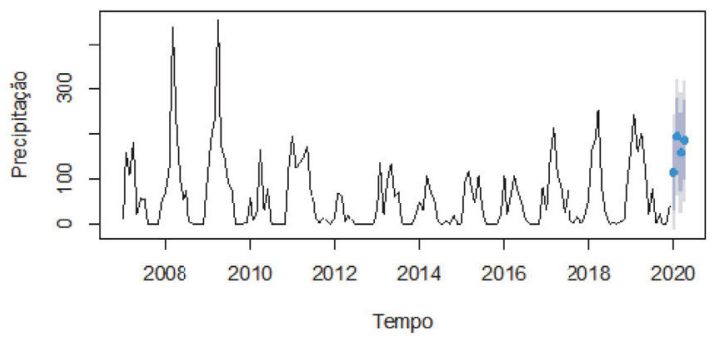

D - Dengue: previsão Holt-Winters Aditivo

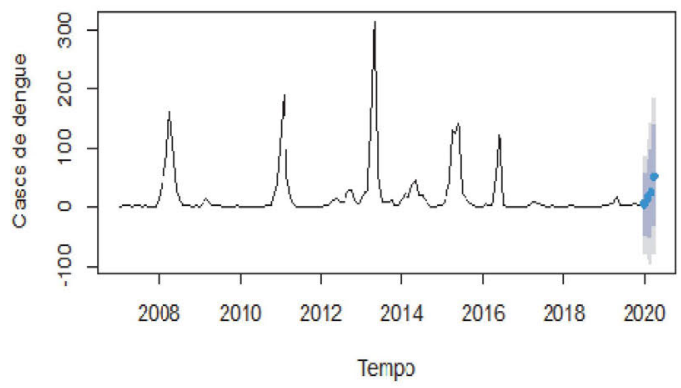


Logo, a previsão estipulou a continuação de chuvas dentre da normalidade para a cidade (Fig. 8-B). No entanto, o erro residual foi alto negativamente em $2010 \mathrm{e}$ 2012 e positivamente em 2016 e 2017, foram meses do ano que não seguiram o padrão da sazonalidade.

No tocante aos casos de Dengue, os valores são muito baixos, com exceção do final de 2015 para o início de 2016, na qual há um pico de notificações em relação aos demais anos da pesquisa na cidade (Fig. 8-D).

A previsão (Fig. 8-D) seguiu o padrão dos anos da pesquisa com dados de ocorrências baixos, não ultrapassando 15 casos mensais. Portanto, a previsão foi fidedigna à tendência apresentada no período dos anos do estudo. Em relação ao erro residual, este está conectado ao pico de 2015, o que reflete aos valores negativos até o final de 2016.

\subsubsection{Brejo Santo}

A previsão ficou dentro do padrão da precipitação dos anos anteriores (Fig. 9-B), principalmente, dos anos secos a normais, seguindo a tendência de dados do período da pesquisa, que mostrou uma tendência de maior quantidade de chuvas nos últimos anos, porém, ainda abaixo do observado até 2008 .

\section{A- Precipitação: decomposição da série temporal}

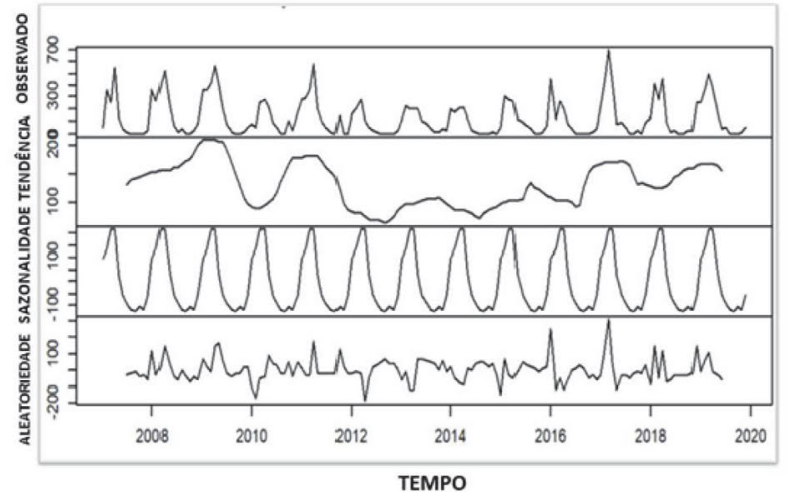

C - Dengue: decomposição da série temporal

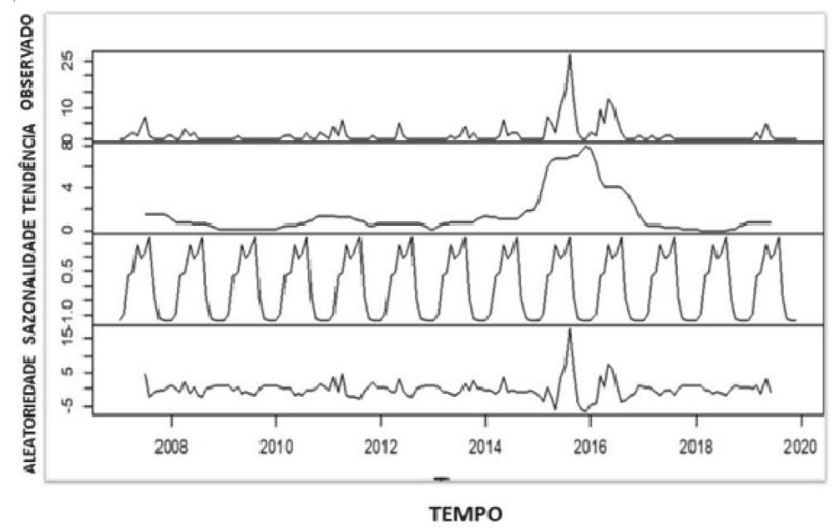

Com relação aos casos de dengue, há uma sazonalidade na série com um considerável aumento de 2014 a 2018 e a partir desse ano uma queda nas notificações.

A previsão para os casos de Dengue (Fig. 9-D) estabeleceu um aumento dos casos em relação aos anos de 2018 e 2019. O erro residual teve valores extremos negativos a partir de 2015 , portanto, a tendência de aumento dos casos influenciou nos resultados do modelo.

No geral, os resultados obtidos através da modelagem contemplaram a expectativa do modelo ser aplicável em dados que apresentam tendência e sazonalidade significativas. Portanto, o modelo confirmou ser adequado, principalmente, para a previsão das precipitações, todavia precisa de ajustes para a previsão dos casos de Dengue.

Essa modelagem mostrou resultados satisfatórios para casos de Dengue em outras pesquisas como Shamsuddin et al. (2016) que analisou os casos de Dengue no Estado de Johor na Malásia através do Holt-Winters e os resultados previram 13 semanas de queda gradual dos casos de Dengue. O referido autor concluiu pela sua pesquisa que o modelo é capaz de capturar o padrão dos casos de Dengue.

Da mesma forma, Buczak et al. (2018) através do seu estudo sobre métodos conjuntos para previsão da Dengue compreendeu que o modelo Holt-Winters foi um

\section{B- Precipitação: previsão Holt-Winters Aditivo}

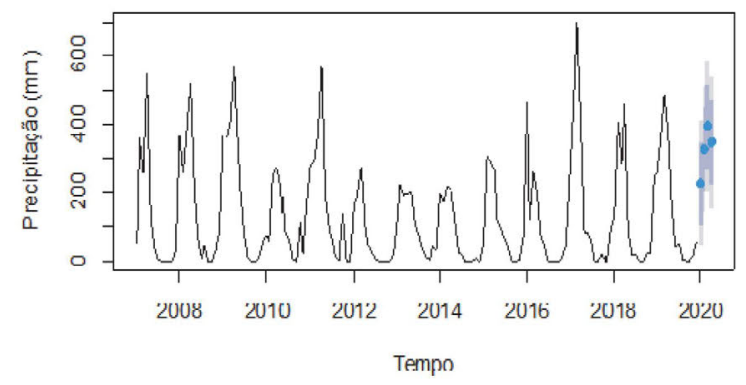

\section{D- Dengue: previsão Holt-Winters Aditivo}

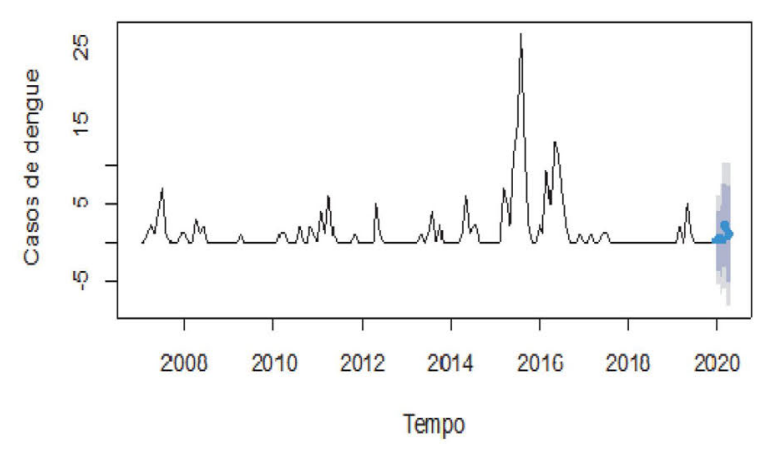

Figura 8 - Resultados da modelagem para casos de dengue em Viçosa do Ceará (2007-2019). 
A- Precipitação: decomposição da série temporal

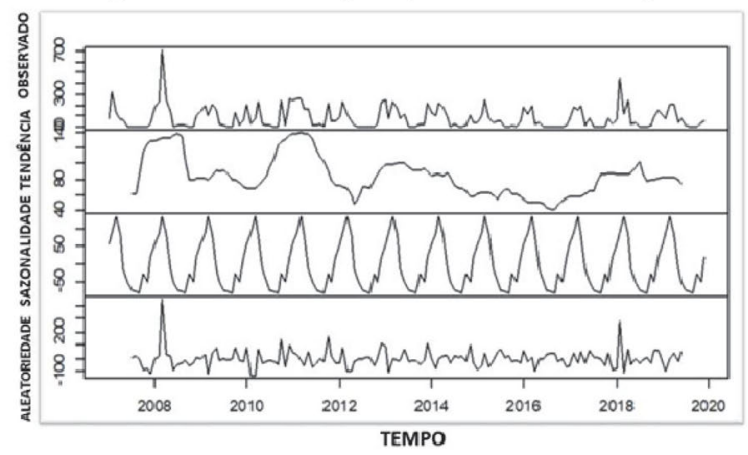

C - Dengue: decomposição da série temporal

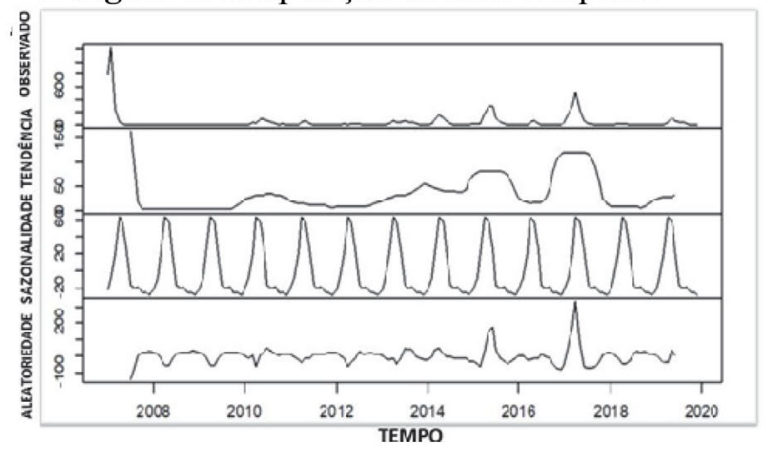

B- Precipitação: previsão Holt-Winters Aditivo

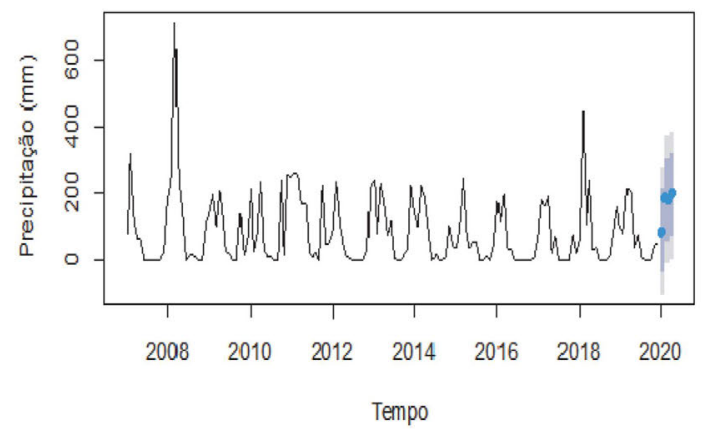

D- Dengue: previsão Holt-Winters Aditivo

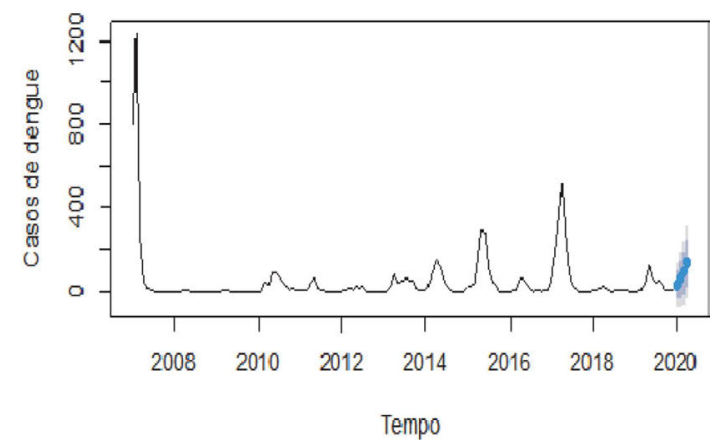

Figura 9 - Resultados da modelagem para os casos de dengue em Brejo Santo (2007-2019).

modelo muito sensível aos diferentes períodos de sazonalidade e aos pontos finais dos testes.

\section{Conclusões}

Os casos extremos da doença aconteceram, majoritariamente, em anos caracterizados como secos para cada cidade e as menores ocorrências em anos chuvosos ou muito chuvosos, exceto Aquiraz, onde as duas variáveis predominaram em anos de chuvas consideradas dentro da normalidade.

Durante o período de atuação da La Niña, existem, aproximadamente, $80 \%$ de chance dos anos serem considerados normais a muito chuvosos incluindo os quatro municípios da pesquisa, levando em consideração os últimos 13 anos. Em contrapartida, referente aos anos com a presença do El Niño, $50 \%$ das vezes os anos foram caracterizados como seco ou muito seco e ao acrescentar os anos normais, essa taxa foi para $85 \%$.

Para Boa Viagem, Viçosa do Ceará e Brejo Santo, nos anos mais secos registraram-se mais ocorrências de casos de Dengue. Para Aquiraz, os anos normais apresentaram mais casos da doença.

O município com maior pluviosidade no período da pesquisa foi Viçosa do Ceará que também obteve menos casos da Dengue. A cidade com menor pluviosidade foi Boa Viagem e Brejo Santo a cidade com mais ocorrências de casos de Dengue no período de 2007 a 2019.
Os resultados da modelagem foram satisfatórios para as precipitações, tendo em vista que os valores foram muito próximos do observado dos anos anteriores. Portanto, a modelagem através do Holt-Winters Aditivo pode ser usada tanto para analisar quanto predizer as chuvas.

Vale ressaltar que a pesquisa considerou uma variável de forma mais aprofundada: precipitação. Logo, outras variáveis, influenciam em anos com maior ou menor quantidade de casos como as variáveis socioeconômicas e planejamento urbano. Portanto, este estudo não teve como finalidade abarcar a totalidade das explicações para os casos da doença e sim aprofundar a análise de uma variável, no intuito de ser mais uma ferramenta de estudo para compreensão da realidade, bem como uma ferramenta de aplicabilidade para ações de planejamento ao combate da Dengue.

\section{Referências}

ARAÚJO, R.A.F. Utilização de Modelos Matemáticos/Estocásticos na Avaliação da Ocorrência de Dengue em Função do Clima na Paraíba. Tese de Doutorado em Meteorologia, Universidade Federal de Campina Grande, Campina Grande, 107 p., 2012.

ARBOVIROSES. Disponível em: https://www.arca.fiocruz.br/ handle/icict/24607. Acesso em: 21 ago. 2021.

BANDITVILAI, S; ANANSATITZIN, S. Comparative study of three time series methods in forecasting dengue hemor- 
rhagic fever incidence in thailand. In: 35th International Academic Conference. Barcelona, p. 56-63, 2017. Disponível em: https://ideas.repec.org/p/sek/iacpro/6409199. html. Acesso em: 12 mar. 2021.

BARBOSA, R.F. Influência de Variáveis Meteorológicas na Ocorrência de Doenças Infecciosas em Diferentes Localidades de Alagoas. Dissertação de Mestrado em Meteorologia, Universidade Federal de Alagoas, Maceió, 96 p., 2007.

BRASIL, Ministério da Saúde. Disponível em: https://saude. gov.br/. Acesso em: 12 jun. 2020.

BUCZAK, A.L; BAUGHER, B.; MONIZ, L.J.; BAGLEY T.; BABIN, S.M; GUVEN, E. Ensemble method for dengue prediction. PLOS ONE, n. 13, p. 1-23, 2018.

INSTITUTO DE PESQUISA E ESTRATÉGIA ECONÔMICA DO CEARÁ. IPECE. Disponível em: https://www.ipece. ce.gov.br/perfil-municipal-2017/. Acesso em: 22 abr. 2019.

LUZ, K. Dados laboratoriais e atualização em vacinas contra a dengue. In: Painel Multidisciplinar Dengue, p. 23-26. 2014. Disponível em: https://www.medlink.com.br/sites/ default/files/artigos/dengue/painel-multidisciplinar-dengue-2014.pdf. Acesso em: 22 out. 2019.

MAGAlHÃES, G. B. Comportamento Espaço-Temporal da Dengue e sua Relação com os Elementos Atmosféricos e Socioeconômicos em Fortaleza/CE. Tese de Doutorado em Geografia, Universidade Federal do Ceará, Fortaleza, 264 p., 2014.

MENDONÇA, F.; DANNI-OLIVEIRA, I.M. Climatologia: Noções Básicas e Climas do Brasil. São Paulo: Oficina de Textos, 2007.

Olivoto, T.; SARI, B.G. Software R Para Avaliação de Dados Experimentais: Um Foco em Experimentos Agronômico, v. 2. 2021.

R DEVELOPMENT CORE TEAM. R: A Language and Environment for Statistical Computing. R Foundation for Statistical Computing. Disponível em: http://www.Rproject.org. Acesso em: 12 jan. 2021.

RIBEIRO, H. (Org.). Geoprocessamento e Saúde: Muito Além de Mapas. Barueri, SP: Manole, 2017.

SHAMSUDDIN, R.M.; RAHMAN, N.A.; RAHMAN, S.A.S.A. Analysis of Dengue Cases In Johor State, Malaysia. In: Proceedings of the 1st Master Project Symposium on Systems Engineering and Professional Science, p. 28-34. 2017. Disponível em: https://razak.utm.my/master-project/ wp-content/uploads/sites/234/2016/02/Proceedings-1stMP-SEPS-PartA.pdf\#page=35. Acesso em: 12 mar. 2021 .

SILVA, N.S. Avaliação da Relação Entre a Climatologia, as Condições Sanitárias (Lixo) e a Ocorrência de Arboviroses (Dengue e Chikungunya) em Quixadá-CE no Período Entre 2016 e 2019. Dissertação de Mestrado Profissional em Climatologia, Universidade Estadual do Ceará, Fortaleza, 97 p., 2019.

SYLVESTRE, G. Biologia do mosquito vetor da dengue e mecanismos de controle. In: Painel Multidisciplinar Dengue, p. 26-32. 2014. Disponível em: https://www.medlink.com. br/sites/default/files/artigos/dengue/painel-multidisciplinardengue-2014.pdf. Acesso em: 22 out. 2019.

TEIXEIRA, M.G. Situação epidemiológica da dengue no mundo e no Brasil e perspectivas durante a Copa 2014. In: Painel Multidisciplinar Dengue, p. 04-09. 2014. Disponível em: https://www.medlink.com.br/sites/default/files/artigos/dengue/painel-multidisciplinar-dengue-2014.pdf. Acesso em: 22 out. 2019.

XAVIER, T.M.B.S.; XAVIER, A.F.S. Papel da componente meridional do vento na costa do Nordeste para a previsão da chuva no Estado do Ceará. In: Anais $1^{\circ}$ Simpósio Brasileiro de Recursos Hídricos, Vitória, p. 537-543, 2004.

License information: This is an open-access article distributed under the terms of the Creative Commons Attribution License (type CC-BY), which permits unrestricted use, distribution and reproduction in any medium, provided the original article is properly cited. 